\title{
Case - Spontaneous thrombosis of varix in childhood
}

Joshua Quisias ${ }^{1}$; Ernest Pang Chan ${ }^{2}$; Timothy Liu Miao ${ }^{3}$; Alvaro Ramirez ${ }^{2}$; Ian Ross ${ }^{3}$; Peter Zhan Tao Wang ${ }^{2}$

${ }^{1}$ Schulich School of Medicine and Dentistry, Western University, London, ON, Canada; ${ }^{2}$ Division of Urology, Department of Surgery, Schulich School of Medicine and Dentistry, Western University, London, ON, Canada; ${ }^{3}$ Department of Medical Imaging, Schulich School of Medicine and Dentistry, Western University, London, ON, Canada

Cite as: Quisias J, Chan EP, Miao TL, et al. Case - Spontaneous thrombosis of varix in childhood. Can Urol Assoc J 2021 February 12; Epub ahead of print.

http://dx.doi.org/10.5489/cuaj.7016

Published online February 12, 2021

$* * *$

\section{Introduction}

A varicocele occurs when there is abnormal enlargement of the pampiniform plexus. In a study of 4,052 boys aged 2-19 years old, overall prevalence was $7.2 \%$, with most cases occurring in boys $>13$ years. ${ }^{1}$ The prevalence was $<1 \%$ in boys under 10 years. ${ }^{1}$ The diagnosis is made on physical examination, but can be confirmed by ultrasonography (US) with demonstration of dilation of the pampiniform plexus ( $>2-3 \mathrm{~mm}$ in diameter). Varicoceles are classified as primary or secondary. ${ }^{2}$ Primary varicoceles are the result of insufficient drainage of the internal spermatic vein due to incompetent valves. Secondary varicoceles are due to compression of the spermatic vein by intra-abdominal processes, such as hydronephrosis, retroperitoneal masses, or 'nutcracker syndrome'. 3

Left untreated, varicoceles can lead to testicular atrophy, scrotal pain, and infertility. The most common indication for surgical intervention for a varicocele in adults is male-factor infertility. ${ }^{6}$ Most men with varicoceles, however, are able to achieve paternity without intervention. ${ }^{3,5}$ In children, the most common indications for surgical intervention are scrotal pain, undesired cosmesis, or significant testicular size asymmetry. ${ }^{7}$ Other indications for intervention include impaired testosterone production and progressive testicular failure. ${ }^{5}$

Thrombosis of a varicocele is exceptionally rare. No guidelines exist for management of this diagnosis, but some case reports have shown effective management with conservative and/or surgical approaches. ${ }^{8-11}$ In this report, we describe a case of unilateral thrombosed varicocele in a child managed conservatively. 


\section{Case report}

A four-year-old boy was referred urgently to the pediatric urology outpatient clinic for suddenonset scrotal swelling. This was associated with mild scrotal discomfort, but no pain or tenderness. There was no history of testicular trauma, urinary tract infection, or changes to voiding. He was born at term with normal growth and was otherwise healthy. There was no family history of hematological disorders.

On physical exam, the left hemi-scrotum had a large, dark blue-hued tubular structure that was soft to palpation (Figure 1). This structure was separate from and much larger than either testis. The right hemi-scrotum showed no abnormalities. Both testes were normal to palpation.

Scrotal US performed two days prior revealed a large, serpiginous, tubular structure within the left hemi-scrotum appearing separate from the left testis and corresponding to the palpable abnormality, most likely representing a large, tortuous left-sided varicocele (Figure 2). This measured up to $2 \mathrm{~cm}$ in diameter and approximately $4.5 \times 4.0 \times 2.5 \mathrm{~cm}$ in overall dimensions. The lesion was predominantly anechoic but contained echogenic material and significantly diminished flow on Doppler interrogation, suggesting thrombosis of the varicocele. Both testes and epididymides appeared normal. Abdominal US was also performed, which revealed no abdominal mass or obstructing lesion. A repeat scrotal US performed two weeks later showed slight interval enlargement of the varicocele measuring up to $5.3 \mathrm{~cm}$. Internal flow was no longer appreciable, suggesting progressive thrombosis.

The patient was admitted for further work up. MRI of the abdomen and pelvis confirmed a large and tortuous thrombosed left-sided varicocele, without involvement of the left spermatic cord or testicular vein (Figure 3). Again, there was no evidence of an abdominal mass or obstructing lesion. Serum electrolytes, lactate dehydrogenase, renal function, coagulation studies, and tumour markers were all within reference range. Pediatric hematology noted that anticoagulation was not indicated due to unremarkable coagulation studies. He was discharged after an uncomplicated two-day stay with instructions for outpatient follow-up.

Subsequent follow-up visits found that the patient continued to be asymptomatic with progressive resolution of the varicocele (Figure 2); however, it remained palpable at 10 months post-discharge. Follow-up scrotal US revealed progressive decrease in size of the varicocele, with largest dimension of $1.5 \mathrm{~cm}$. At two years follow-up, there was full clinical resolution with no asymmetry of testicular size on US.

\section{Discussion}

Spontaneous thrombosis of a varicocele is an exceptionally rare phenomenon. Scrotal US is the imaging modality of choice for further evaluation of varicocele; if the appearance is typical, testicular size can be followed by US to assess for developing asymmetry. If the varicocele size is large, the appearance suggests thrombosis, or in the case of an isolated right-sided varicocele, further imaging may be performed to exclude an obstructing retroperitoneal mass. In children, 
US of the abdomen and pelvis can screen for such masses. If thrombosis of a varicocele is suggested on US, confirmation with cross-sectional imaging may be performed with MRI (preferred over computed tomography in pediatric patients due to the lack of ionizing radiation), which can also aid in the exclusion of an obstructing mass. Spermatic venography is generally reserved for endovascular, or occasionally surgical treatment. ${ }^{12}$

Thromboses in pediatric populations are often iatrogenic (e.g., intravenous catheter) or due to a predisposing condition or factor (e.g., malignancy, infection, immobility). ${ }^{13}$ Thrombotic disorders should be considered in the context of a family history of thrombosis at $<50$ years old, thrombosis without a clear inciting factor, or recurrent thrombosis. ${ }^{13}$ The most common congenital thrombotic disorder is activated protein $\mathrm{C}$ resistance, which is due to a mutation in the Factor $\mathrm{V}$ gene. ${ }^{13}$ Of the acquired thrombotic disorders, antiphospholipid syndrome is the most common. ${ }^{13}$ This condition can be primary or secondary (e.g., due to systemic lupus erythematosus). In the case described in this report, a predisposing thrombotic condition is unlikely given the absence of contributory family history or recurrent thrombotic events and normal coagulations studies. It was felt by pediatric hematology that this child should not be susceptible to further thrombotic events.

Four cases of thrombosed varix in adults have been described.$^{8-11}$ Unlike the case reported here, each of these cases presented with acute scrotal pain. ${ }^{8-11}$ Two cases underwent varicocelectomy after failure of medical management ${ }^{8,9}$ while the others were managed with analgesics, anti-inflammatories, and anticoagulants. ${ }^{10,11}$ In the case described by Kamel et al. (2018), the patient was placed on enoxaparin and evaluated for hematologic abnormalities. A protein $\mathrm{C}$ deficiency was discovered, and the patient remained on anticoagulation with acenocoumarol. $^{10}$

Coolsaet and Weinberg (1980) reported three cases of thrombosis of the spermatic vein in children aged 7, 10, and 15. ${ }^{14}$ Each report was associated with acute scrotal pain and swelling. One patient had a history of similar symptoms, which was managed by orchidopexy for suspected testicular torsion. Upon symptom recurrence, he was diagnosed with thrombosis of the spermatic vein by venography and managed medically with anti-inflammatories. The other two cases underwent surgical exploration through an inguinal incision. One was managed with antiinflammatory medications, and the other underwent excision of the thrombosed veins to the level of the external ring. There was no recommendation for hematological work-up.

\section{Conclusions}

In the setting of thrombosed varix in children without pain or evidence of intraabdominal compression, thrombotic disorders, or hemodynamic instability; a conservative approach may be taken with serial clinical and radiographic follow-up. Use of anti-inflammatory medications can be considered in patients with discomfort or without resolution over time. The absence of pain may be due to lack of significant compression of nearby neural fibers. ${ }^{15}$ A hematology referral should be made to assess for disorders of thrombosis (e.g. protein $\mathrm{C}$ resistance or 
antiphospholipid syndrome). Varicocelectomy can be considered in cases refractory to medical management. 


\section{References}

1. Akbay E, Çayan S, Doruk E, et al. The prevalence of varicocele and varicocele-related testicular atrophy in Turkish children and adolescents. BJU International 2000;86:49093.

2. Bhosale $\mathrm{P}$, Patnana $\mathrm{M}$, Viswanathan $\mathrm{C}$, et al. The inguinal canal: anatomy and imaging features of common and uncommon masses. RadioGraphics 2008;28:819-35.

3. Sack B, Schäfer M, Kurtz M. The Dilemma of Adolescent Varicoceles: Do They Really Have to Be Repaired?. Curr Urol Rep 2017;18.

4. Cheungpasitporn W, Horne J, Howarth C. Adrenocortical carcinoma presenting as varicocele and renal vein thrombosis: a case report. J Med Case Rep 2011;5.

5. Vakalopoulos I, Kampantais S, Lymperi S, et al. Should we expand the indications for varicocele treatment?. Transl Androl and Urol 2017;6:931-42.

6. Kroese AC, de Lange NM, Collins J, et al. Surgery or embolization for varicoceles in subfertile men. Cochrane Database Syst Rev 2012;10:CD000479.

7. Macey M, Owen R, Ross S, et al. Best practice in the diagnosis and treatment of varicocele in children and adolescents. Ther Adv Urol 2018;10:273-82.

8. Amador Robayna A, Rodríguez Talavera J, Ballesta Martínez B, et al. Deep vein thrombosis: a rare cause of acute testicular pain. Case report: literature review. Urol Int 2018;101:117-20.

9. Raghavendran M, Venugopal A, Kiran Kumar G. Thrombosed varicocele - a rare cause for acute scrotal pain: a case report. BMC Urol 2018;18.

10. Kamel K, Gassen S, Mohamed M, et al. Bilateral spontaneous thrombosis of the pampiniform plexus; a rare etiology of acute scrotal pain: a case report and review of literature. Afr J Urol 2018;24:14-18.

11. Ouanes Y, Sellami A, Chaker K, et al. Thrombosis of the pampiniform plexus: about a case report. Urol Case Rep 2018;20:28-29.

12. Gendel V. Antegrade pampiniform plexus venography in recurrent varicocele: case report and anatomy review. World J Radiol 2011;3:194.

13. Rodriguez V, Warad D. Pediatric coagulation disorders. Pediatr Rev 2016;37:279-91.

14. Coolsaet B, Weinberg R. Thrombosis of the spermatic vein in children. J Urol 1980;124:290-91.

15. Paick S, Choi W. Varicocele and testicular pain: a review. World J Mens Health 2019;37:4. 


\section{Figures and Tables}

Fig. 1. Left: Large left scrotal mass with blue-hued tubular structure present. Photo taken three days after onset of swelling. Right: Resolution of left thrombosed varix 16 months after initial presentation.

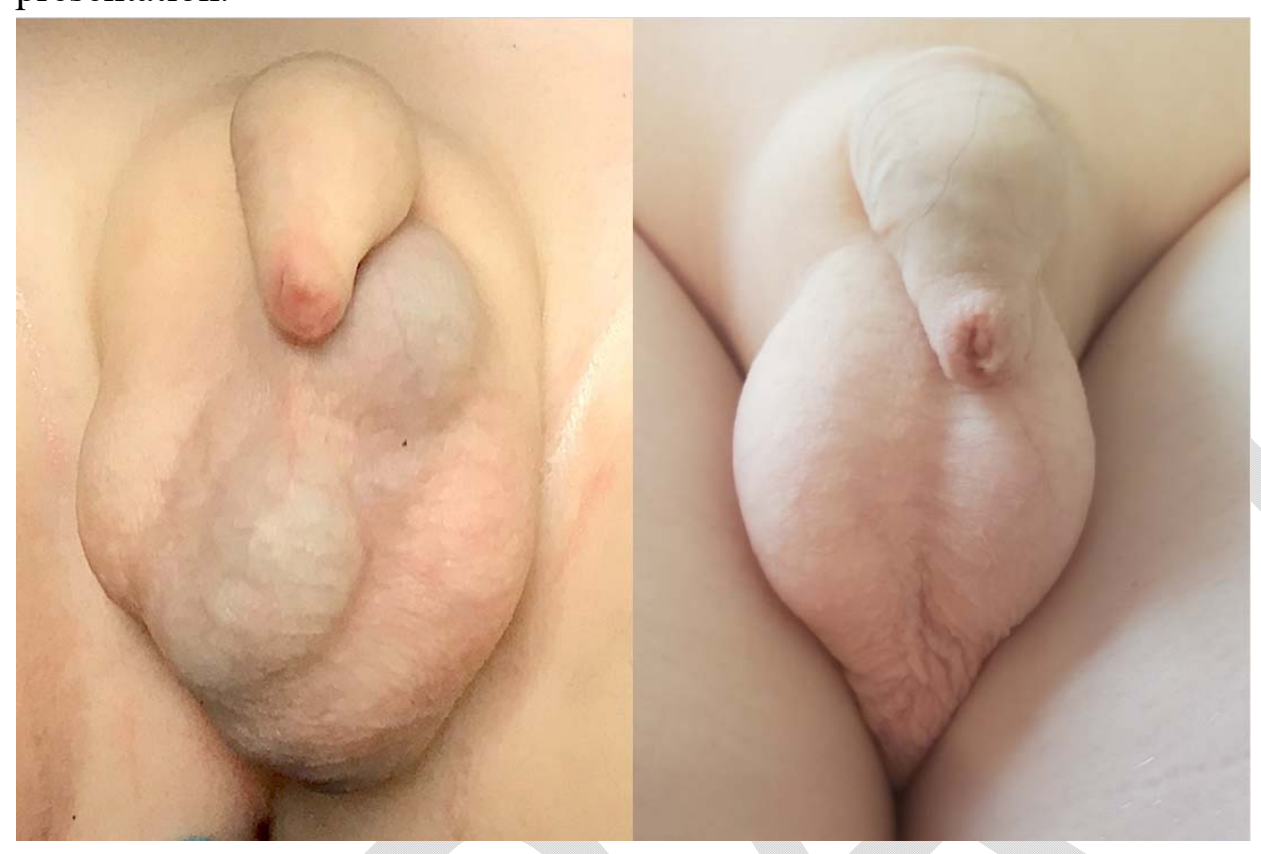

Fig. 2. Transverse ultrasound of the left hemi-scrotum demonstrates a tortuous, predominantly anechoic tubular structure separate from the normal left testis (T). This contained internal echogenic material and significantly diminished flow (not shown), highly suggestive of a thrombosed left-sided varicocele (V).

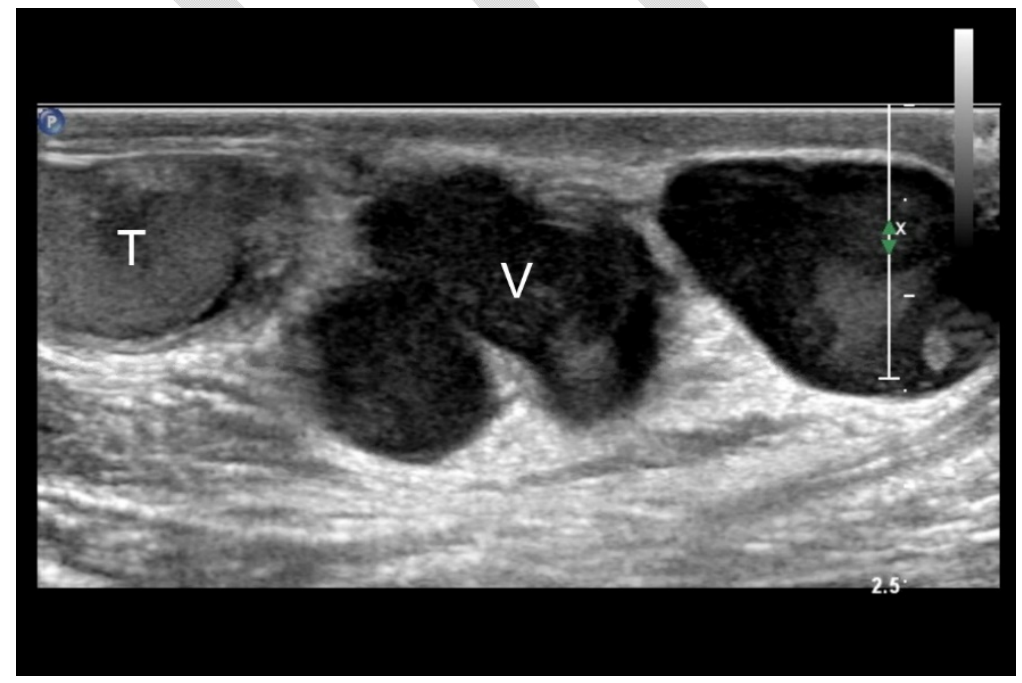


Fig. 3. Coronal contrast-enhanced $\mathrm{T} 1$ weighted image from subsequent magnetic resonance imaging (MRI) of the abdomen and pelvis confirms a dilated left varicocele with thick enhancing walls and increased intraluminal signal, reflecting thrombosis. The remainder of the examination was normal, with no evidence of an obstructing mass.

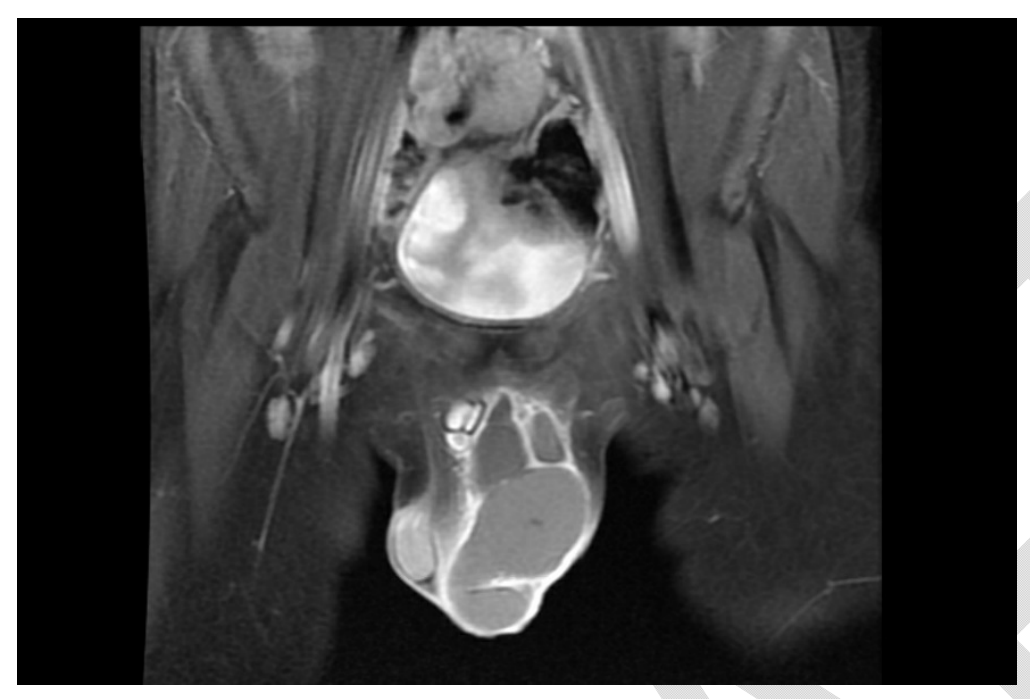

